\title{
LA TRAITE DE JEUNES FEMMES MIGRANTES SUBSAHARIENNES EN TUNISIE: ENTRE DROITS GARANTISSANT LA DIGNITÉ HUMAINE ET RÉALITÉ TRAGIQUE
}

\author{
TRATA DE MUJERES MIGRANTES SUBSAHARIANAS EN TÚNEZ: \\ ENTRE LOS DERECHOS PARA GARANTIZAR LA DIGNIDAD HUMANA \\ Y LA REALIDAD TRÁGICA \\ THE TREATMENT OF YOUNG SUBSAHARIAN MIGRANT WOMEN \\ IN TUNISIA: BETWEEN RIGHTS GUARANTEEING HUMAN DIGNITY \\ AND TRAGIC REALITY
}

NAIMA FEKIH*

\section{RESUME}

Fondé sur une étude qualitative basée sur la méthode de Focus Groupe auprès des jeunes femmes migrantes subsahariennes habitant au grand Tunis, nous assistons à un regain d'intérêt à la problématique de traite de jeunes femmes migrantes subsahariennes en Tunisie. Notre objectif est d'étudier les motifs de migration ainsi que la situation de jeunes femmes migrantes en Tunisie en employant une étude quantitative basée sur la technique de Focus Groupe. Notre étude a bien montré l'écart entre droits tunisiens garantissant la dignité humaine et réalité tragique rencontrée par ces migrantes en Tunisie à cause de la traite. La traite apparaît comme un crime qui évolue de façon remarquable dès la révolution tunisienne, et malgré les procédures juridiques et les différentes initiatives adoptées dans le but de combattre ce phénomène, la traite persiste jusqu'à l'heure actuelle, ce qui nécessite la mise en place d'une stratégie innovante permettant de faire face à ce phénomène.

MOTS- CLÉS: la traite, les jeunes femmes migrantes subsahariennes, droits, dignité humaine, réalité tragique.

\begin{abstract}
RESUMEN
Sobre la base de un estudio cualitativo basado en el método del Grupo de enfoque entre mujeres jóvenes migrantes subsaharianas que viven en el Gran Túnez, estamos presenciando un interés renovado en el problema de la trata de mujeres jóvenes migrantes subsaharianas en Túnez. Nuestro objetivo es estudiar los motivos de la migración y la situación de las jóvenes migrantes en Túnez mediante un estudio cuantitativo basado en la técnica de Focus Group. Nuestro estudio mostró claramente la brecha entre los derechos tunecinos que garantizan la dignidad humana y la realidad trágica que enfrentan estas mujeres migrantes debido a la trata, que parece ser un crimen que está evolucionando de manera notable desde la revolución, y a pesar de los procedimientos legales y las diversas iniciativas adoptadas para combatir este fenómeno, persiste hasta ahora, lo que requiere una estrategia innovadora para enfrentar este fenómeno.
\end{abstract}

PALABRAS CLAVE: trata, jóvenes migrantes subsaharianas, derechos, dignidad humana, realidad trágica.

* Doctorat en démographie. Faculté des sciences humaines et sociales. Université de Tunisie. BP 113 douz 4260 Kebilli, Tunisie.

naimafekih6@gmail.com 


\section{ABSTRACT}

Based on a qualitative study based on the Focus Group method among young sub-Saharan migrant women living in Greater Tunis, we are witnessing a renewed interest in the problem of trafficking of young sub-Saharan migrant women in Tunisia. Our aim is to study the reasons for migration as well as the situation of young migrant women in Tunisia by using a quantitative study based on the Focus Group technique. Our study clearly showed the gap between Tunisian rights guaranteeing human dignity and the tragic reality faced by these migrant women in Tunisia because of trafficking. Trafficking appears to be a crime that is evolving in a remarkable way since the Tunisian revolution, and despite the legal procedures and the various initiatives adopted to combat this phenomenon, the trafficking persists until now, which requires the in place of an innovative strategy to deal with this phenomenon.

KEYWORDS: trafficking, young sub-Saharan migrant women, rights, human dignity, tragic reality.

\section{INTRODUCTION}

La traite des migrants est un phénomène qui se trouve dans tous les pays de ce monde, qu'ils soient pays d'origine, de transit ou de destination. Ce crime affecte la Tunisie qui est une région de fortes dynamiques migratoires internationales caractérisées par le passage d'une migration classique masculine à une participation élevée des femmes dans cette mobilité.

La Tunisie, Dès la révolution du 14 Janvier 2011, devenait un pays d'immigration et une société multiculturelle où la diversité des origines des migrants atteint un niveau sans précédent. Les conditions misérables de vie dans le pays d'origine amènent un nombre important de jeunes femmes subsahariennes à faire le choix de la migration régulière ou irrégulière, seules ou à l'aide de réseaux criminels, pour arriver à la Tunisie, dans le but d'être créatrices de leur destin. De ce fait, la mobilité apparaît comme un véritable moyen pour accéder à un statut social et économique respectable et à une sécurité abandonnée dans leur pays natal. Malgré les efforts déployés par le gouvernement Tunisien, à travers les procédures juridiques diversifiées, pour protéger les ressortissants venus de l'étranger, les jeunes femmes subsahariennes se trouvent dans une situation de fragilité en subissant des formes de violences et de traite aux fins d'exploitation de travail forcé, d'exploitation sexuelle au profit de réseaux criminels organisés.

Notre étude exploratrice marquera une nouvelle étape dans les recherches qualitatives sur la traite de jeunes femmes migrantes subsahariennes arrivées en Tunisie qui reste un thème mal connu. Cette mobilité de jeunes femmes subsahariennes en tant qu'expérience sociale est elle un facteur d'intégration et d'accès aux droits garantissant la dignité humaine et le bien- être social ou simplement un facteur d'exploitation et de traite?

Cette recherche offrira des pistes de réflexion sur le thème en accordant une 
importance particulière à l'expérience sociale vécue par les jeunes femmes migrantes subsahariennes en Tunisie pour connaitre si la migration est un moyen pour promouvoir la situation et la sécurité ou juste une aventure qui conduit à une vie misérable reflète un paradoxe entre droits mis de la part du gouvernement Tunisien visant la protection des migrants et une réalité plus tragique basant sur la traite qui énonce la violation des droits de ces migrants. En plus, à travers une recherche empirique approfondie, ce travail essaye d'éclairer le phénomène de traite de jeunes femmes subsahariennes en Tunisie, pour arriver à proposer les solutions possibles permettant de rendre la Tunisie un pays d'accueil qui pourra offrir les conditions convenables aux étrangers sans violations de leurs droits.

Notre étude, a bien démontré que la Tunisie est concernée par le problème de traite de jeunes femmes migrantes subsahariennes en tant que pays d'accueil. Les secteurs d'exploitations et de traite varient, de même que le profil des trafiquants, ils vont de l'esclavage domestique à l'implication forcée dans des activités criminelles, en passant par le travail et la mendicité forcés et en plus l'exploitation sexuelle.

La situation fragile de jeunes femmes migrantes subsahariennes affirment que malgré la mise en place d'une loi relative à la prévention et la lutte contre la traite des personnes (loi N61- 2016) qui a vu le jour en août 2016 et une instance nationale de lutte contre la traite des personnes qui a été mise en place en Février, le problème de traite persiste jusqu'à nos jours et les migrantes restent menacées par cette infraction, ça veut dire qu'il reste un nombre de défis à relever en Tunisie, pour lutter efficacement le phénomène de traite qui est en progression remarquable en affectant une hiérarchie sociale vulnérable.

Notre travail est structuré en cinq parties: Méthodologie adoptée, le cadre conceptuel, les raisons de la migration vers la Tunisie pour les jeunes femmes migrantes subsahariennes, la problématique de la traite chez les jeunes femmes migrantes subsahariennes, l'expérience Tunisienne dans la lutte de la traite des migrants. En conclusion de ce travail, un ensemble de recommandations a été formulé à l'attention des acteurs-clés pour bien combattre la traite et protéger les migrantes subsahariennes qui sont recensées jusqu'à présent comme victimes de ce crime dans notre société Tunisienne.

\section{MÉTHODOLOGIE ADOPTÉE}

Ce travail s'appuie sur une enquête qualitative sur la base de Focus group, menée dans le grand Tunis durant les mois d'octobre -novembre en 2017, est suivie par une recherche complémentaire durant les deux mois Janvier -Février 2019, au- 
près d'un groupe formé de 25 migrantes subsahariennes âgées entre 15 et 25 ans, et un nombre des acteurs sociaux clefs telles que les membres de la société civile, des personnels de santé, des citoyens, des responsables de l'état, etc, ayant contact et expérience en relation étroite avec l'objet de cette étude. Ces Focus group étaient centrés sur la situation de jeunes femmes migrantes subsahariennes en Tunisie et les différentes facettes de traite vécue et en essayant de découvrir à quel degré les procédures juridiques réussissent à combattre la traite de jeunes femmes migrantes qui reste jusqu'à nos jours une question large. Lors de nos investigations, nous remarquons la forte préoccupation auprès des jeunes femmes migrantes subsahariennes autour leur situation qui reste menacée par la traite malgré toutes les procédures juridiques adoptées dans notre pays. Nos résultats de recherche sur terrain sont complétés par une revue littérature sur la question: rapports et études, articles de presse, etc.....

Le tableau ci-dessous présente d'une manière très succincte les profils des jeunes femmes migrantes enquêtées:

Tableau 1. Les profils des jeunes femmes subsahariennes

\begin{tabular}{|c|c|c|}
\hline \multicolumn{2}{|c|}{ Variable } & $\mathbf{F}$ \\
\hline \multirow{2}{*}{ Âge } & $15-24$ ans & 18 \\
\hline & 25 et + & 7 \\
\hline \multirow{4}{*}{ Nationalité } & Ivoirienne & 10 \\
\hline & Camerounaise & 8 \\
\hline & Burkinabè & 3 \\
\hline & Congolaise & 4 \\
\hline Religion & Chrétien & 25 \\
\hline \multirow{3}{*}{ Niveau d'instruction } & Primaire & 12 \\
\hline & Secondaire & 5 \\
\hline & Supérieur & 8 \\
\hline \multirow{4}{*}{ Statut professionnel } & Occupé-non déclaré & - \\
\hline & Sans emploi & 10 \\
\hline & Etudiant & 8 \\
\hline & Autre & 7 \\
\hline \multirow{2}{*}{ Statut matrimonial } & Célibataire & 15 \\
\hline & Marié & 10 \\
\hline \multirow{3}{*}{ Statut de séjour } & Avec papiers & 12 \\
\hline & Sans papiers & 8 \\
\hline & Refugié & 5 \\
\hline Total & \multicolumn{2}{|l|}{25} \\
\hline
\end{tabular}

Source: Propre élaboration. 
La plupart des enquêtées appartenant à la tranche d'âge 15-24 ans avec un pourcentage de $72 \%$, et le reste (28\%) ont l'âge 25 ans et plus. Ces jeunes femmes ont des nationalités différentes; la majorité est ivoiriennes et Camerounaises (72\%) et les restes ont la nationalité Burkinabé (12\%) ou Congolaise (16\%). Concernant le niveau scolaire ; la majorité ont cessé leur scolarité au niveau primaire (48\%), au niveau secondaire pour certains (20\%), alors que les autres au niveau supérieur (32\%). 40\% des femmes interviewées sont en chômage, alors que $32 \%$ de l'échantillon sont des étudiantes et $28 \%$ exercent des activités différentes. La plupart des femmes enquêtées (60\%) sont célibataires alors que (40\%) d'elles sont mariées. Pour le statut de séjour, la majorité des interviewées existent en Tunisie avec leurs papiers (48\%) ça veut dire en situation régulière, alors que $32 \%$ d'elles sans papiers et le reste (20\%) sont des réfugiées, ça veut dire $52 \%$ de ces femmes interviewées sont en situation irrégulière.

\section{LE CADRE CONCEPTUEL}

\subsection{Définition de la traite des migrants}

Selon l'étude exploratoire réalisée par l'OIM en 2013 (OIM, 2017, p.16) sur la traite des personnes en Tunisie d'une part, et les résultats de notre travail sur terrain avec les femmes migrantes subsaha- riennes d'autre part, la Tunisie est un pays source, de destination et de transit des victimes de traite, parmi ces victimes apparaissent les femmes et les hommes d'origine subsaharienne (Afrique de l'Ouest).

De son côté, la loi Tunisienne considère la traite des personnes comme "L'attirement, le recrutement, le transport, le transfert, le détournement, le rapatriement, l'hébergement ou l'accueil des personnes, par le recours ou la menace de recours à la force ou aux armes ou à toute autre forme de contrainte, d'enlèvement, de fraude, de tromperie, d'abus d'autorité ou d'une situation de vulnérabilité ou par l'offre ou l'acceptation de sommes d'argent ou avantages ou dons ou promesses de dons afin d'obtenir le consentement d'une personne ayant autorité sur une autre aux fins d'exploitation, quelle qu'en soit la forme, que cette exploitation soit commise par l'auteur de ces faits ou en vue de mettre cette personne à la disposition d'un tiers". Cette définition est inspirée de l'article 3 du protocole de Palerme (OIM, 2019, p.8)

La traite et la migration sont étroitement liées. Certes, l'état de vulnérabilité des migrants est souvent exploité par les trafiquants pour exercer le processus de traite. Ainsi, la traite des migrants est l'une des plus graves violations des droits humains et une forme moderne d'esclavage. Les migrants en tant que victimes sont exploités dans les travaux 
domestiques, la construction, le travail forcé dans l'agriculture, la mendicité, la prostitution forcée, la restauration, l'exploitation sexuelle, etc. Et selon quelques témoignages recueillis lors de nos investigations, la traite apparaît comme un crime contre l'humanité, une pratique consiste à isoler et menacer les migrants afin de mieux les priver de leurs droits et de leurs libertés et les exploiter sans donner aucune importance à leur dignité humaine ou à leur bien- être social.

\subsection{Les approches de l'étude}

Dans le but de bien comprendre et expliquer le phénomène étudié, nous allons utiliser un nombre des approches. Certes, aucune approche théorique ne peut être jugée supérieure aux autres car, ces approches sont liées et complémentaires en abordant une réalité multidimensionnelle qui est la migration féminine et notamment la migration des femmes subsahariennes vers la Tunisie.

\subsubsection{L'approche du choix rationnel (TCR): George Homans (1961)}

Selon cette approche les individus sont vus comme des acteurs qui choisissent au mieux de leurs intérêts entre des alternatives, même si des contraintes et des structures restreignent les choix possibles (Haug, 2008). La recherche a néanmoins ultérieurement complexifié l'image d'une personne migrante rationnelle qui chercherait uniquement à maximiser son utilité. Julian Wolpert
(1965) décrit ainsi un acteur qui chercherait à «satisfaire» un besoin et non pas à le «maximiser». La recherche d'une destination migratoire prendrait ainsi fin une fois une telle destination acceptable trouvée, sans nécessairement que l'ensemble des destinations possibles soient examinées (Étienne Piguet, 2013, p.143).

\subsubsection{L'approche par le genre}

Mirjana Morokvasic (1984), rappelle que la migration est également féminine. L'apport de Morokvasic porte essentiellement sur la diversité des destins des femmes migrantes à travers le monde en illustrant des nombreux cas d'exploitation de cette main-d'œuvre. Selon elle, la migration féminine peut être positive (émancipation, autonomie financière), mais elle peut aussi renforcer les inégalités de sexe et menacer les femmes migrantes à travers les dangers rencontrés (Mirjana Morokvasik, 2011, p.11).

\subsubsection{L'approche de "place utility» et "Residentiel Stress»: Julian Wolper}

Le concept de «place utilty» joue, à nos jours un rôle important dans plusieurs recherches et études sur les processus de décision migratoire. Les migrations sont considérées comme résultant d'une série de facteurs de «stress». Les théories de la satisfaction résidentielle n'ont pas assez pris en considération les caractéristiques spécifiques qui lient les personnes à l'endroit où elles habitent et génèrent ou atténuent le niveau de «stress résidentiel» (la somme des facteurs de tous ordres qui contribuent à une éventuelle 
insatisfaction) (Deane, 1990). Cet élargissement conceptuel consiste en somme à mieux prendre en considération les caractéristiques des lieux de départ et d'arrivée et leur appréhension par les individus (Étienne Piguet, 2013, p.145).

\section{POURQUOI LES JEUNES FEMMES SUBSAHARIENNES MIGRENT VERS LA TUNISIE?}

Á nos jours, la migration touche tous les pays, et les femmes constituent environ 50\% de la population migrante (FMMD, 2016, p.3), tandis que durant les années
70 et 80 , elles ne représentent que $10 \%$ (OIM). Les récits des unes et des autres font dégager que la migration est le résultat de facteurs d'impulsion caractérisant les pays d'origine et de facteurs d'attraction caractérisant la Tunisie en tant que pays de destination. Migrer est, un acte qui peut être réalisé de façon volontaire ou forcée, de façon régulière ou irrégulière, de manière individuelle ou dans le cadre d'un groupe. Nous détaillons dans le tableau ci- dessous les différents facteurs des mouvements migratoires mentionnés par les jeunes femmes interviewées :

Tableau 2. Les facteurs de migration des jeunes femmes subsahariennes

\begin{tabular}{|c|c|}
\hline $\begin{array}{l}\text { Les facteurs d'impulsion } \\
\text { Pays d'origine }\end{array}$ & $\begin{array}{l}\text { Les facteurs d'attraction } \\
\text { Pays de destination «La Tunisie» }\end{array}$ \\
\hline $\begin{array}{l}\text { - Le chômage } \\
\text { - La perte d'emploi } \\
\text { - La pauvreté et manque d'opportunités } \\
\text { - Les conditions sociales misérables } \\
\text { - Les troubles politiques : les guerres, l'insécurité } \\
\text { - les situations de violences, de viol, de violation des droits } \\
\text { humains } \\
\text { - La faiblesse des mesures de protection sociale } \\
\text { - La discrimination et la redistribution inégale des ressources } \\
\text { - La dégradation de l'environnement } \\
\text { - Les catastrophes naturelles }\end{array}$ & $\begin{array}{l}\text { - Les opportunités de travail, d'études et de promouvoir les } \\
\text { carrières (les sportives exemple) } \\
\text { - La bonne qualité de l'éducation } \\
\text { - La sécurité } \\
\text { - Les disparités économiques entre le pays d'origine et la } \\
\text { Tunisie } \\
\text { - L'existence de réseau migratoire constitué d'amis, de pro- } \\
\text { ches, de membre de la famille ou des connaissances ayant } \\
\text { déjà émigrés à la Tunisie } \\
\text { - L'accès facile à l'information (les réseaux sociaux, la télévi- } \\
\text { sion, migrants, etc.) joue un rôle dans la construction d’un } \\
\text { monde idéal au-delà des frontières } \\
\text { - La curiosité et l'envie de découvrir la Tunisie }\end{array}$ \\
\hline
\end{tabular}

Source: Propre élaboration.

Les facteurs de migration sont liés, pour les jeunes femmes migrantes subsahariennes, à la nécessité de fuir le monde misérable et la situation de fragilité et de vulnérabilité. Elles choisissent la Tunisie comme un refuge, un lieu favorable où les droits humains sont protégés et les opportunités sont multiples en permettant d'espérer une véritable amélioration des conditions de vie. Il ressort que la migration constitue un projet de vie individuel, familial et même collectif.
Migrer, est un processus qui résulte des facteurs notés précédemment. Certes, la réussite de certaines femmes migrantes est une source inspirant d'autres, celles qui vivent le même rêve, à prendre la même destination, et à affronter les mêmes dangers. La migration est une culture par laquelle sont conçus le futur des unes et les destins des autres. 
Figure 1. Schéma récapitulatif du processus migratoire chez les jeunes femmes subsahariennes
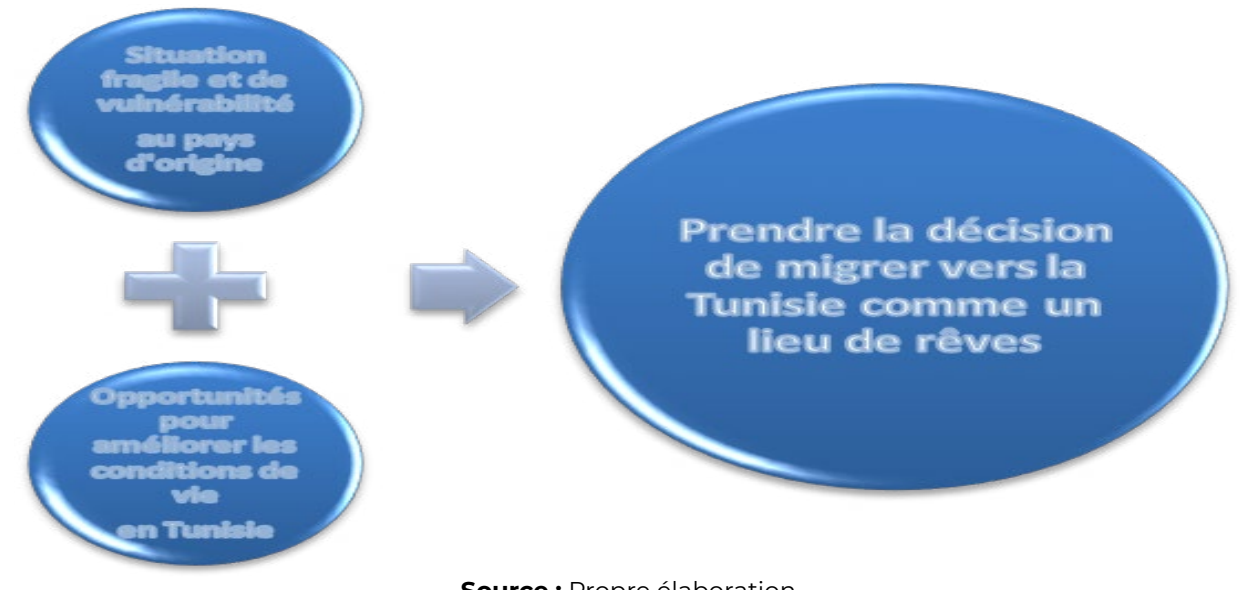

Source : Propre élaboration

\section{LA PROBLÉMATIQUE \\ DE LA TRAITE CHEZ LES JEUNES FEMMES MIGRANTES}

\subsection{Les jeunes femmes subsahariennes de l'espoir vers la traite}

La vie dans les pays d'origine pour les jeunes femmes subsahariennes est une réalité dure et misérable. Nombreuses parmi elles cherchent et espèrent une vie ailleurs qui répond à leurs aspirations, et lorsqu'elles ne trouvent pas la chance par eux- mêmes, elles acceptent sans hésitation les propositions qui leur sont faites pour arriver en Tunisie, et pour accéder à un travail, ou aux études, ou à d'autres situations permettant d'atteindre les objectifs espérés. Les témoignages des femmes interviewées affirment que leur espoir se transforme en un cauchemar dès qu'elles quittent leurs pays d'origine. Elles deviennent victimes qu'il soit des trafiquants lors de leur passage de leurs pays vers la Tunisie ou d'exploiteurs dans le pays d'accueil. (Manuel de formation sur la lutte contre la traite, 2009)

Selon plusieurs études les migrants internationaux en général sont de plus en plus exposés à la traite et la violation de leurs droits par les exploitants et les fraudeurs. Ces dernières années et notamment depuis 2013 les statistiques montrent bien que le phénomène de traite est en évolution. L'OIM Tunisie, a détecté 594 cas, 93\% des victimes sont originaires de la Côte d'Ivoire, 2.5\% du Nigeria, $2 \%$ du Sénégal, et $2.5 \%$ d'autres nationalités, parmi ces victimes $82 \%$ sont des femmes. (OIM, 2019)

Il ressort de notre étude que les jeunes femmes migrantes subsahariennes deviennent vulnérables à cause de la migration qui donne la chance aux trafiquants d'exploiter cette hiérarchie sociale dans divers domaines qu'on peut les citer dans le tableau ci-dessous : 
Tableau 3. Les domaines D'exploitation des jeunes femmes subsahariennes

\begin{tabular}{|c|c|}
\hline Le domaine d'exploitation & Nombre \\
\hline Le travail domestique & 10 \\
\hline Le travail agricole forcé & 2 \\
\hline La restauration & 4 \\
\hline La mendicité & 3 \\
\hline La prostitution et l'exploitation sexuelle & 6 \\
\hline Total & 25 \\
\hline
\end{tabular}

Source : Propre élaboration.

Les déclarations des unes et des autres font dégager que les jeunes femmes migrantes subsahariennes n'avaient pas conscience des risques et des menaces éventuels qu'elles pouvaient affronter durant leur séjour en Tunisie. Le rêve de migration n'entrave pas le voyage, et à posteriori elles découvrent et vivent les difficultés, les risques et la traite mais la situation diffère d'une migrante à une autre. La plupart (40\%) sont exploitées dans le travail domestique sans rémunération ou avec très bas prix, elles ne jouissent pas d'une relation contractuelle et d'une couverture sociale, d'autres (24\%) sont exploitées dans la prostitution et l'exploitation sexuelle, alors qu'un nombre d'entre elles est exploité dans la restauration (16\%), et (12\%) des interviewées sont exploitées dans la mendicité, et le reste (8\%) sont exploitées dans le travail agricole forcé.

Figure 2. Les domaines D'exploitation des jeunes femmes migrantes subsahariennes

Les domaines d'exploitation des jeunes femmes migrantes subsahariennes

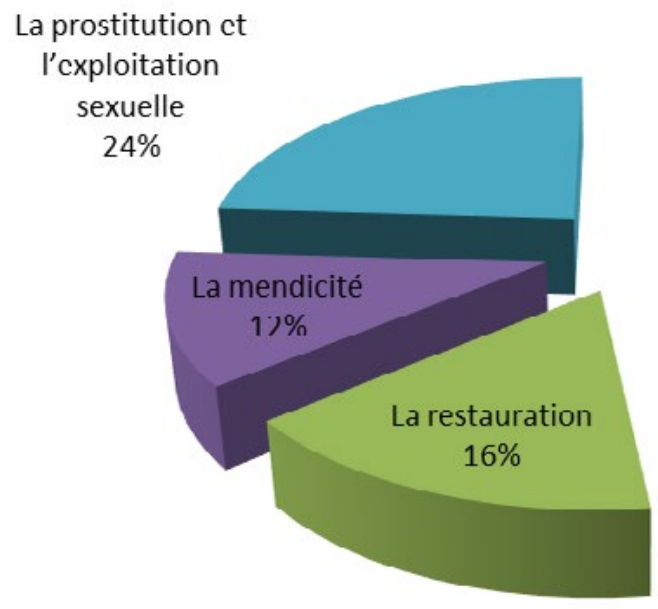

Source: Propre élaboration.

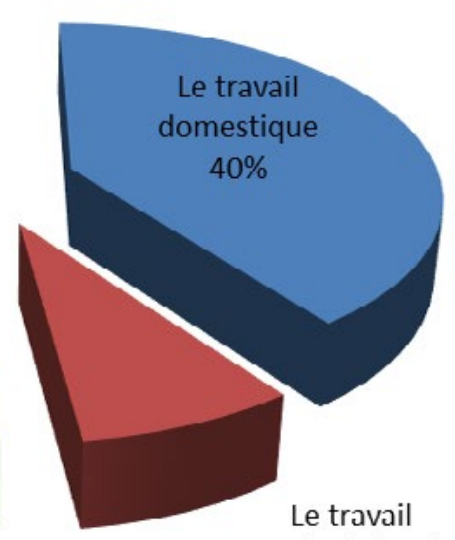

agricole forcé

$8 \%$ 
Certes la majorité des femmes rencontrées sont dans une situation irrégulière en Tunisie, n'ayant pas des cartes de séjour, elles consentent sans hésitation à des propositions et promesses attractives dans le but d'améliorer leurs conditions de vie. Les recruteurs profitent de la crédulité et de la vulnérabilité de ces femmes pour réaliser des gains financiers. La situation professionnelle délicate et la condition sociale misérable affirment que les migrantes sont considérées comme des marchandises. Ce statut impacte la vie des jeunes femmes migrantes qui devenait plus précaire, tragique et traumatisante en rendant les migrantes plus fragiles surtout avec un futur ambigu et des horizons fermés.

L'une des jeunes femmes a déclaré: «J'ai accepté le travail chez la famille pour retrouver un revenu et satisfaire mes besoins en Tunisie sans connaître les contraintes qui seront exercées sur moi, j'ai travaillé dans des conditions inacceptables, sans rémunération, ni couverture sociale, juste j'ai eu le droit de manger, boire et dormir, en plus que ça j'ai été privée de toute liberté de mouvement, je n'ai pas eu le droit de sortir, de voir le monde. Et puisque ma situation était irrégulière en Tunisie je n'ai pas pu rien faire, j'ai subi de servitude domestique deux ans. Le recruteur m'a considéré comme étant marchandise et chaque fois il m'a vendu à une famille dans le but d'avoir plus des profits, j'ai vécu la traite en sentant la mort chaque
seconde...Malgré les droits en Tunisie les migrantes souffrent jusqu'à nos jours....» (K. 22 ans. Cameroun)

Notre étude montre que dès l'entrée en Tunisie les recruteurs ou les trafiquants contraignent les jeunes femmes migrantes à travers la tromperie, les fausses promesses, la force ou en abusant de leur vulnérabilité à accepter la servitude avec ses différentes facettes. La situation de vulnérabilité à cause la situation irrégulière, la pauvreté ou autres causes, rendre les jeunes femmes migrantes dépendantes matériellement ou autrement des exploitants sans être conscientes la situation dans laquelle elles se trouvent, elles acceptent de se soumettre à l'exploitation et la violence qui sont exercés sur elles. (Géorgie, article 3) Ces situations d'exploitation et de violence s'agissent d'une situation de traite et les migrantes sont des victimes de traite quelle que soit leur situation juridique.

\subsection{Les effets de la traite}

Le phénomène de la traite des jeunes femmes migrantes a des effets néfastes sur les migrantes elles-mêmes et sur la société Tunisienne dans sa totalité.

\subsubsection{Les impacts sur les jeunes femmes migrantes}

La traite met la vie des jeunes femmes migrantes en danger en menaçant l'état physique et psychologique. Les conséquences sont multiples et se différent selon le type et le degré de l'exploitation, parmi les on peut citer: 
- Les violences à l'égard des jeunes femmes migrantes résultent des blessures, des fractures et parfois le handicap physique ou mental et même la mort.

- Les grossesses non désirées pour les jeunes femmes migrantes victimes d'exploitation sexuelles ou pour les jeunes femmes travailleuses domestiques.

- Les jeunes femmes migrantes ont des difficultés pour accéder aux soins, elles se trouvent dans des conditions de vie et d'hygiène misérable, elles devenaient malades (hépatites, VIH/ Sida, etc).

- La situation de traite est stressante pour les victimes, elle résulte le traumatisme, les troubles, la dépression, la manque d'estime de soi, l'isolement, les ruptures des liens avec la famille et les autres, le sentiment d'insécurité d'exclusion et de marginalisation. Les jeunes femmes migrantes sentent qu'elles sont des marchandises, personnes privées de leurs droits, de leurs libertés, de leur vie personnelle et professionnelle, elles sentent un état de tiraillement, elles existent comme des corps sans sensation c'est la mort blanche qui conduit dans plusieurs cas au suicide.

\subsubsection{Les impacts sur la société Tunisienne}

- Certes, les réseaux de criminalité organisée qui sont derrière la traite des jeunes femmes migrantes conduisent à l'augmentation de violence, de corruption, de criminalité et de terrorisme, ce qui menace la sécurité dans notre pays.

- Les menaces sur l'économie nationale à la suite de l'accroissement de marchés parallèles, l'économie informelle.

- La difficulté d'accès des jeunes femmes migrantes aux services de santé, sociales ou d'éducation résulte des effets néfastes en matière de santé publique et de protection sociale

- Le recul de la cohésion sociale à cause de la discrimination, de l'injustice et de l'intolérance envers les jeunes femmes migrantes.

\section{L'EXPÉRIENCE TUNISIENNE DANS LA LUTTE DE LA TRAITE DES MIGRANTS}

\subsection{Les procédures juridiques: Droit Tunisien garantissant la dignité humaine}

La constitution de la Tunisie est la première garantie des droits de la personne et de la dignité humaine (OIM, Manuel sur la loi relative à la prévention et à la lutte contre la traite des personnes en Tunisie, 2017, p18). L'État garantit aux citoyens et aux étrangers les libertés et les droits, comme il donne une importance à l'égalité entre les sexes et la lutte contre la violence basée sur le genre à travers la promulgation de la loi N58- 2017 de la lutte de la violence à l'égard de femme qui entre en vigueur en 2018. 
Dès la ratification du protocole de Palerme ${ }^{1}$ en 2013 (Décret N 2003-698), la Tunisie criminalise la traite des personnes et notamment celle des migrants et s'engage à lutter contre ce phénomène en adoptant des mesures législatives nécessaires.

La Tunisie adopte la loi N 61-2016 du 3 août 2016 relative à la prévention et à la lutte contre la traite des personnes. Cette initiative permettra à la Tunisie de prévenir toutes les formes d'exploitation auxquelles pourraient être soumises les migrantes, protéger et assister les migrants en tant que victimes, poursuivre les auteurs de la traite et développer un partenariat à l'échelle nationale et internationale dans le domaine de la lutte contre la traite des personnes et notamment les migrants.

\subsection{Stratégie des $4 \mathrm{P}$ et le rôle de l'instance nationale de lutte contre la traite des personnes}

La Tunisie comme les autres pays qui ont ratifié le protocole de Palerme, élabore la stratégie nationale des $4 \mathrm{P}$ de lutte contre la traite des personnes qui repose sur quatre éléments:

- Prévention: prévenir la traite des personnes par le biais d'un plan d'action national contre ce phénomène sous toutes ses formes en adoptant des politiques publiques favorables à la protection des migrants en tant que grou-

\footnotetext{
Le protocole de Palerme additionnel à la convention des Nations Unies contre la criminalité transnationale organisée.
}

pes vulnérables. Dans le but de bien connaitre le phénomène il est nécessaire selon la loi de rassembler d'informations et développer de statistiques sur les victimes, ensuite il faut impliquer les parties prenantes dans la lutte contre la traite dans le but de réduire les causes de vulnérabilité qui peuvent conduire aux situations de traite telle que la pauvreté, l'exclusion sociale, la criminalité, etc. Ces parties doivent promouvoir l'éducation des jeunes, pour connaitre l'importance du respect des droits des autres, surtout les migrants et découvrir la gravité des phénomènes de discrimination, de violence traite dans notre société.

- Protection: Protéger et assister les victimes de la traite. La loi de la lutte contre la traite donne une importance particulière à la victime qui a le droit d'être protégée et assistée selon les besoins. La lutte contre la traite exige le respect et la protection des droits des victimes y compris les migrants, les demandeurs d'asile, et les réfugiés. Le Schémas ci- dessous mentionne les droits de la victime: 


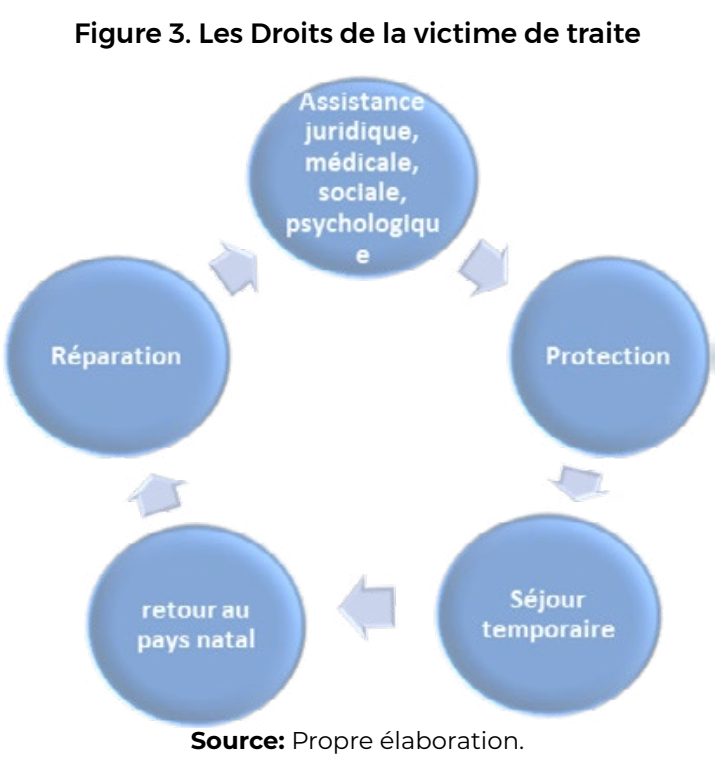

Les migrantes en tant que victimes de la traite doivent être traitées sans discrimination et avec précaution par tous les intervenants et toutes les instances qui seront impliqués dans leur assistance. Dès l'identification des migrantes victimes quel que soit leur statut juridique en Tunisie, ces dernières doivent être informées de leurs droits et des différents moyens d'assistance qu'elles peuvent recevoir sans oublier d'estimer les risques à toutes les étapes d'assistance afin de garantir la sécurité et la protection de toutes les personnes impliquées.

La loi garantit aux victimes la traite de façon individuelle en fonction de leurs besoins et leurs circonstances. Les mesures d'assistance sont préparées au cas par cas en tenant compte de l'âge des femmes migrantes victimes et leurs besoins spécifiques, en traitant les réactions de ces victimes avec toute compréhension et tolérance. Les types d'assistance sont:
- Assistance médicale: La prise en charge et les soins gratuits dans les établissements publics de santé.

- Assistance Juridique: Indemnisation et l'aide judiciaire.

- Assistance sociale et psychologique: Soutien psychologique et social, prise en charge, réinsertion sociale, hébergement.

- Autres mesures telles que le retour volontaire, droit de séjour dans les cas déterminés par la loi, offrir les informations nécessaires pour les femmes migrantes étrangères avec la langue que les victimes comprennent.

La loi de lutte contre la traite donne un intérêt particulier aux victimes étrangères tout en facilitant leur retour volontaire à leurs pays natals compte tenu de leur sécurité, et dans le cas des risques dans leurs pays, les migrantes auront le séjour permanent en tant que réfugiées en bénéficiant de la protection internationale. 
- Poursuite: Poursuivre pénalement les auteurs de la traite

- Partenariats: Promouvoir les partenariats avec la société civile, les organisations gouvernementales et les organisations internationales pour lutter efficacement contre la traite des personnes en tant que grave infraction et atteindre les objectifs relatifs aux Protection, Prévention et Poursuite. La coopération aux niveaux national et international, est attribuée à l'instance nationale de lutte contre la traite des personnes.

Dans le but de bien pratiquer la loi de lutte contre la traite, la Tunisie a créé en 2018 l'instance nationale de lutte contre la traite des personnes y compris les migrants, placée sous tutelle du ministère de la justice, et dont la mission est de:

- Coordonner les politiques publiques en matière de lutte contre la traite à l'échelle nationale.

- Mettre en œuvre la stratégie nationale de lutte et prévenir contre la traite et d'assister les victimes.

- Assister les victimes à travers la constitution et le suivi des dossiers auprès des pouvoirs publics en collaboration avec la société civile pour lever les contraintes pouvant entraver l'accès des victimes à leurs droits tels que l'obtention de l'aide judiciaire.

- Promouvoir les coopérations sur le plan international

- Faire des formations et superviser les programmes de formation aux niveaux national et international.

- - Effectuer des études et des recherches dans le but de moderniser les législations.

\subsection{Le rôle de L'OIM et les initiatives de la société civile}

L'organisation internationale pour les migrations (OIM) travaille en collaboration avec ses partenaires de la communauté internationale dans le but de résoudre les problèmes des migrants notamment la traite, en offrant des procédures de protection physique et l'assistance nécessaire aux migrantes victimes de la traite. Et en partenariat avec l'instance nationale de la lutte contre la traite et la société civile l'OIM engage des actions de sensibilisation ciblant les migrantes en tant que groupes vulnérables pour connaitre leurs droits et comment protéger eux-mêmes de la traite à travers les différentes procédures juridiques, en plus, l'OIM s'efforce pour la réinstallation de ces victimes dans un nouveau cadre de vie.

L'OIM et la société civile jouent un rôle important dans le soutien des procédures juridiques, ce qui aide à promouvoir la dignité humaine et le bien- être des jeunes femmes migrantes.

\section{CONCLUSION}

L'intérêt pour la question de traite des jeunes femmes migrantes en Tunisie était, 
jusqu'à une époque récente, rare et exceptionnel. Dès la révolution Tunisienne, et avec l'accroissement des actes d'exploitation des jeunes femmes migrantes subsahariennes, ce sujet constitue l'une des préoccupations primordiales qu'il soit pour l'état, les organisations internationales ou la société civile. Il n'était sans doute inévitable que les initiatives pour la prévention, l'assistance des migrantes en tant que victimes de traite, et le respect de leurs droits humains, réagissent face à un sujet très sensible pour la Tunisie sur le plan politique et social. Les procédures juridiques à travers la promulgation de la loi de lutte contre la traite des personnes et la création de l'instance nationale de lutte contre la traite, et les initiatives de la société civile et le rôle de l'OIM, aident à donner une importance au sujet de traite des migrantes en adoptant les démarches possibles pour soutenir cette hiérarchie sociale. En revanche, la vulnérabilité et la fragilisation des jeunes femmes migrantes persistent, et la traite reste en accroissement remarquable.

La criminalisation et la pénalisation de la traite des jeunes femmes migrantes en Tunisie ne suffisent pas pour faire face à cette forme moderne d'esclavage. Les résultats recueillis affirment que la violation des droits des femmes migrantes persiste jusqu'à nos jours surtout avec la continuité des facteurs favorisant la traite et la fragilisation de ces migrantes.
Certes, la traite des jeunes femmes migrantes constitue un crime contre ces migrantes d'une part, et contre l'État d'autre part, ce qui nécessite d'autres solutions plus efficaces. Il est important de corriger les carences en matière de législations, connaissances, formation, intervention, et suivie auprès des différents intervenants dans la lutte contre la traite des jeunes femmes migrantes subsahariennes.

Notre recherche nous offre l'occasion pour mentionner des pistes d'actions qui pourraient être adoptées dans le but de combattre la traite des jeunes femmes migrantes en garantissant leur sécurité d'une part et la paix dans notre pays d'autre part:

- La mise en place des poursuites et des sanctions plus sévères permet d'avoir un effet dissuasif sur les futurs auteurs de traite et les trafiquants.

- Inciter les femmes migrantes à travers la sensibilisation pour témoigner les actes de traite.

- Le lancement de campagnes de sensibilisation pour le public sur les droits des migrantes et les dangers de traite sur cette hiérarchie sociale et sur notre pays. La sensibilisation aide non seulement à informer le public des dangers de la traite mais aussi à sauver les jeunes femmes migrantes qui la subissent déjà.

- La réalisation des études et des recherches pour rassembler des statis- 
tiques et des données sur la situation des migrantes et découvrir les difficultés qu'elles vivent en Tunisie. La base de données pourra servir à mettre en relation les acteurs sociaux en facilitant leur intervention pour combattre la traite des femmes migrantes.

- Mobiliser les médias et le secteur privé dans la lutte contre la traite des femmes migrantes.

- Servir les réseaux sociaux numériques tels que le Facebook, Twitter, Youtube, à combattre la traite. Ce sont des supports très efficaces pour la sensibilisation, la découverte des cas de traite, l'échange des informations entre les ONG et entre la société civile et l'état, la diffusion des informations sur des initiatives de lutte contre la traite des femmes migrantes etc.

- Éduquer les enfants à travers le processus de socialisation à respecter les droits des femmes migrantes.

- Faire de la traite des femmes migrantes une grave infraction nécessitant des sanctions plus sévères.

- Création d'un observatoire nationale de lutte contre la traite, et qui mettra en place une équipe spécialisée sa mission est la recherche des éventuelles victimes de la traite, la prévention et l'assistance en facilitant la vie des femmes migrantes en Tunisie à travers l'offre des travaux, l'hébergement, l'éducation, la formation, la sensibilisation, la régulation des papiers, etc. Cette démarche empêche l'exploita- tion des jeunes femmes migrantes par les trafiquants. Cet observatoire pourra travailler en collaboration avec l'instance nationale de lutte contre la traite, l'OIM et la société civile.

- Formation des fonctionnaires de police, des agents des services d'immigration, des assistants sociaux, etc dans le but de renforcer leur capacité dans la détection des cas de traite des femmes migrantes et assurer les procédures d'intervention nécessaires.

- Pour aider les jeunes femmes migrantes subsahariennes à se protéger de traite, on doit avoir une vision rigoureuse de ce qui les rend vulnérables, en identifiant les facteurs de fragilisation (les facteurs économiques, sociaux, culturels, politiques et juridiques).

- Les médias tels que le radio, la télévision et la presse constituent des supports efficaces pour faire connaitre et comprendre le phénomène de traite des femmes migrantes subsahariennes à travers la diffusion des informations sur les infractions des droits des migrantes en Tunisie. Ils constituent des moyens de prévention par le biais de la sensibilisation en renforçant le regard humanitaire envers les migrantes subsahariennes. 


\section{RÉFÉRENCES BIBLIOGRAPHIQUES}

BIT. (2009). Manuel de formation sur la lutte contre la traite des enfants à des fins d'exploitation par le travail. Sexuelle ou autres, Genève.

Décret N2003-698 du 25/3/2003. Jort N26 du 1/4/2003.

Deane Glenn, D. (1990). Mobility and adjustments: paths to the resolution of residential stress. Demography, 27(1), 65-79.

Étienne, P. (2013). Les théories des migrations, synthèse de la prise de décision individuelle, Revue Européenne des migrations internationales, vol. 29. N3. 2013, Migrations de retour et de rapatriement, Open Edition Journals, 2013, pp141-161, Université de Poitiers.

FMMD. (2016). Les droits des femmes migrantes une perspective fondée sur les droits de l'enfant. 2ème édition. Document relais 4 . Projet de texte à débattre lors des journées de la société civile du FMMD. Global Forum on Migration Development. Le lien est: https://destination-unknown.org/wp-content/ uploads/BP-4-Rights-of-migrant-women-FR-web.pdf (visité le 10/5/2019)

Géorgie. (2006). Loi relative à la lutte contre la traite des êtres humains. Article 3.

Haug, S. (2008). Migration Networks and Migration Decision-Making. Journal of Ethnic and Migration Studies, 34(4), 585-605. Disponible en: https://globalnetwork.princeton.edu/bellagio/Migration\%20Networks\%20and\%20Migration\%2oDecision-Making.pdf

Mirjana, M. (2011). Migrations et mobilisées, l'invisibilité continue. Cahiers du genre,
N51, 2011, pp 25- 47, Revue Cairn. Info. Le lien: https://www.cairn.info/revuecahiers-du-genre-2011-2-page-25.htm\# . (Révisé le 22/04/2019).

Organisation Internationale pour les migrations (OIM) (2017). Manuel sur la loi relative à la prévention et à la lutte contre la traite des personnes en Tunisie, Tunisie.

OIM. Le lien: www. iom. Int, Global Estimates and Trends, visité le 2/1/2019.

Organisation Internationale pour les migrations (OIM). (2019). Manuel sur la loi relative à la prévention et à la lutte contre la traite des personnes en Tunisie. Tunisie.

OIM Maroc. (2010). Manuel de formation de base, «Agir contre la traite des personnes et le trafic de migrants».

Wolpert, J. (1965). Behavioral aspects of the decision to migrate. University of Pennsylvania.

\section{PARA CITAR ESTE ARTÍCULO}

Fekih, N. (2019). La traite de jeunes femmes migrantes subsahariennes en Tunisie: Entre droits garantissant la dignité humaine et réalité tragique. Collectivus, Revista de Ciencias Sociales, 6(2), 141-157.

DOI: http//dx.doi.org/10.15648/Coll.2.2019.9

Recibido: 01/12/2018 Aprobado:04/03/2019 